\title{
Vocal Quality Assessment of Patients with Pituitary Adenomas Operated via Endoscopic and Microscopic Approaches
}

\author{
Gokhan KURAN1, Gokhan CAVUS² \\ ${ }^{1}$ Adana City Training and Reseach Hospital, Department of Otorhinolaryngology, Adana, Turkey \\ ${ }^{2}$ Adana City Training and Reseach Hospital, Department of Neurosurgery, Adana, Turkey \\ Corresponding author: Gokhan CAVUS gokhanctf@yahoo.com
}

\section{ABSTRACT}

\begin{abstract}
AIM: To evaluate whether there is any difference in voice quality between patients who were operated via microscopic and endoscopic approaches for pituitary adenomas.

MATERIAL and METHODS: The study group consisted of 59 patients and 30 healthy volunteers. They were all males and divided into three groups as; microscopically operated (MO) $(n=30)$, endoscopically operated $(E O)(n=29)$ and control $(n=30)$. Subjective and objective voice analyses were performed.

RESULTS: There was no significant relationship within groups according to Voice Handicap Index-10 ( $p=0.053$ ). Fundamental frequency, jitter \%, jitter PQQ5 parameters revealed that there was a statistically significant difference between $\mathrm{EO}$ and $\mathrm{MO}$ groups. No statistically significant difference was observed within EO, MO and control groups with respect to harmonics-to-noise ratio median scores.

CONCLUSION: The present study assessed the effects of endoscopic and microscopic techniques for removal of pituitary adenomas on quality of voice. As it keeps the anatomically realistic model of sinuses and nasal cavity which provides resonance for the voice, endoscopic intervention found out to be superior by means of vocal quality.
\end{abstract}

KEYWORDS: Pituitary surgery, Endoscopic approach, Microscopic approach, Voice quality

\section{- INTRODUCTION}

$\mathrm{P}$ ituitary adenomas are tumors that generally not cancerous, yet they can cause various symptoms due to distinct hormonal imbalances. These symptoms can occur due to the pressure on tissues nearby the adenomas.

Today, these adenomas are treated with microscopic and endoscopic surgical methods. In 1907, Schloffer has described the transnasal - transsphenoidal approach (3). After the development of endoscopic techniques, Jankowski et al. started to perform endoscopic transsphenoidal pituitary adenoma surgery in 1992 (5). latrogenic changes occur in nasal cavity and sinuses after both methods. Voice is a powerful and impressive communication tool; therefore, sound disturbances affect the individual's life in many ways. According to the current pathology, studies on nasal resonance revealed that sinuses are a significant resonator for sound. It is known that increasing the vocal resonance is one of the functions of nasal cavity and sinuses. Anterior sphenoidotomy is performed as wide as possible in endoscopic surgery. Thus, manuplation of the instruments in the surgical area becomes easier. Although microscopic surgery method is still being used, endoscopic method has become more preferred due to its features like minimal invasiveness, better control over surgical area and shorter recovery periods.

Before 1922, based on his confidence in the transsphenoidal method, Cushing did not hesitate to elaborate on his approach to pituitary tumors in his operative notes. He referred to this route as his customary transphenoidal route. By 1929, 3 years before his retirement as an active surgeon, Cushing gave up the transsphenoidal method and exclusively used 
the subfrontal route, which he referred to as the transfrontal method. In the late 1950s, Guiot proved the effectiveness of transsphenoidal surgery in resection of pituitary tumors and therefore played an important role in reviving the popularity of this method. Later, the introduction of a microscope by Jules Hardy eliminated most of the difficulties encountered during visualization of Cushing's surgical field and transsphenoidal surgery. These pioneers paved the way for the development of the modern techniques that have shaped the current status of transsphenoidal surgery. However, after the 1990s, despite the long learning curve, endoscopic surgery has become widespread due to the development of endoscopic systems, light systems and the advantages of the endoscope (6).

In this study, we assessed whether there is a difference between patients operated with microscopic and endoscopic surgery procedures with respect to vocal quality.

\section{MATERIAL and METHODS}

The Ethics Committee of The Adana City Training and Research Hospital provided ethical approval. The ethic approval number is "AŞH137".

\section{Patients}

This study was conducted on 59 male patients and 30 voluntary males between the years 2017 and 2018 by otorhinolaryngology and neurosurgery clinics in our hospital. Ear-nose-throat examination was performed on all of the patients and volunteers; and nasal cavities together with laryngeal structures were evaluated. None of the patients and volunteers in the study had any sinonasal disturbances. All the patients operated via endoscopic and microscopic surgical routes and were undergone same interventional steps. All microscopic surgeries were performed by a single neurosurgeon (GC), and all endoscopic procedures were performed by a team of the same otorhinolaryngology and neurosurgery specialists (GK and GC). In all patients undergoing endoscopic surgery, the vomer was drilled to increase the field of vision during the surgical procedure. In patients whose nasal septal posterior portion was resected during the procedure, were excluded from the study. In cases with binostril endoscopic approach $(n=4)$, nasal structures were preserved on the side of the endoscope. Standard endoscopic or microscopic transsphenoidal surgeries were performed. The defect occurred during both endoscopic and microscopic surgeries was fixed by using the fascia lata. Patients diagnosed with disorders that block their nasal passage and laryngeal space like sleep apnea, allergic rhinitis, nasal polyposis were also excluded from the study.

Patients were divided into three groups; microscopically operated (MO), endoscopically operated (EO) and control as healthy volunteers. So, the study includes $30 \mathrm{MO}$ patients, 29 EO patients and 30 controls.

Sound analysis was performed in all patients at preoperative period and at postoperative $2^{\text {nd }}$ month.

\section{Subjective Voice Analysis}

Turkish version of Voice Handicap Index (VHI)-10 was used in analyses. This questionnaire includes 10 questions. Each question is rated between 0 to 4 points and the evaluation is made out of 40 points. All three groups completed this questionnaire and the results were assessed.

\section{Objective Analysis}

The Paul Boersma and David Weenink voice analysis system (The Praat) is one of the leading voice analysis programs. All of the patients were performed with voice analysis in a quiet room within $20 \mathrm{~cm}$ distance to microphone (Audio Technica at 2020). During the recording, the patients were told to say the vowel "a" during 3 minutes. The segment analyzed was a 1-s portion in the middle of the vowel production. The selected segments were later digitized (50-kHz sampling rate) and analyzed using the Praat. The fundamental frequency (F0), jitter \%, shimmer \%, shimmer dB, and harmonics-tonoise ratio (HNR) were recorded and the evaluations were statistically compared within groups.

\section{Statistical Method}

Shapiro-Wilk test was conducted for testing the normality of data. In median score comparison of the three groups, OneWay Analysis of Variance (ANOVA), Mean pitch, Jitter \%, Jitter PQQ5, Shimmer \%, Shimmer Db, Shimmer APQ3, Shimmer APQ11, HNR (dB) were used when age of the groups was normally distributed and Kruskal-Wallis $\mathrm{H}$ Test was used when age of the groups was not normally distributed. Mann Whitney $U$ test was used in significant results in median difference detection between two groups. The descriptive statistics of them were expressed by mean, standard deviation, median and by $25^{\text {th }}$ and $75^{\text {th }}$ percentiles. Chi-square test was used in distribution and relationship analysis of $\mathrm{VHI}-10$ categories with respect to groups. All statistical analysis was performed using SPSS v.21.0 (IBM Corp., Armonk, NY, USA). A p value of less than 0.05 was considered as statistically significant.

\section{RESULTS}

Of the 89 subjects, the mean age was $37.99 \pm 9.23$ (range: 18-65) years.

There was no statistically significant relationship within groups with respect to VHI-10 ( $p=0.053)$ (Table I). F0, jitter \%, jitter PQQ5 parameters revealed that there was a statistically significant difference between $\mathrm{EO}$ and $\mathrm{MO}$ groups with respect to their median scores (Figures 1-3).

The values of parameters in $\mathrm{MO}$ group were higher than the EO group. MO group showed an increase with respect to Shimmer \% median scores, but this increase was not statistically significant $(\mathrm{p}=0.450)$.

There was no statistically significant difference within EO, MO and control groups with respect to HNR median scores. All of these evaluations can be seen in Table II.

\section{DISCUSSION}

In pituitary adenomas, endoscopic approach has recently become an important alternative. There are studies that evaluates the size of the tumor, intraoperative and postoperative 
Kuran G. and Cavus G: Vocal Quality and Pituitary Adenomas

Table I: Voice Handicap Index-10 Scores of the Patients and Healthy Volunteers and Their Comparison

\begin{tabular}{lcccc}
\hline $\begin{array}{l}\text { Voice Handicap } \\
\text { Index-10 }\end{array}$ & $\begin{array}{c}\text { Endoscopic } \\
\text { Approach } \\
\mathbf{n}(\%)\end{array}$ & $\begin{array}{c}\text { Microscopic } \\
\text { Approach } \\
\mathbf{n}(\%)\end{array}$ & $\begin{array}{c}\text { Control } \\
\mathbf{n}(\%)\end{array}$ & $\begin{array}{c}\text { Total } \\
\mathbf{n}(\%)\end{array}$ \\
\hline 0 & $24(82.8)$ & $19(63.4)$ & $28(93.3)$ & $71(79.8)$ \\
\hline 1 & $5(17.2)$ & $10(33.3)$ & $2(6.7)$ & $17(19.1)$ \\
\hline 2 & $0(0.0)$ & $1(3.3)$ & $0(0.0)$ & $1(1.1)$ \\
\hline Total & $29(100.0)$ & $30(100.0)$ & $30(100.0)$ & $89(100.0)$ \\
\hline
\end{tabular}

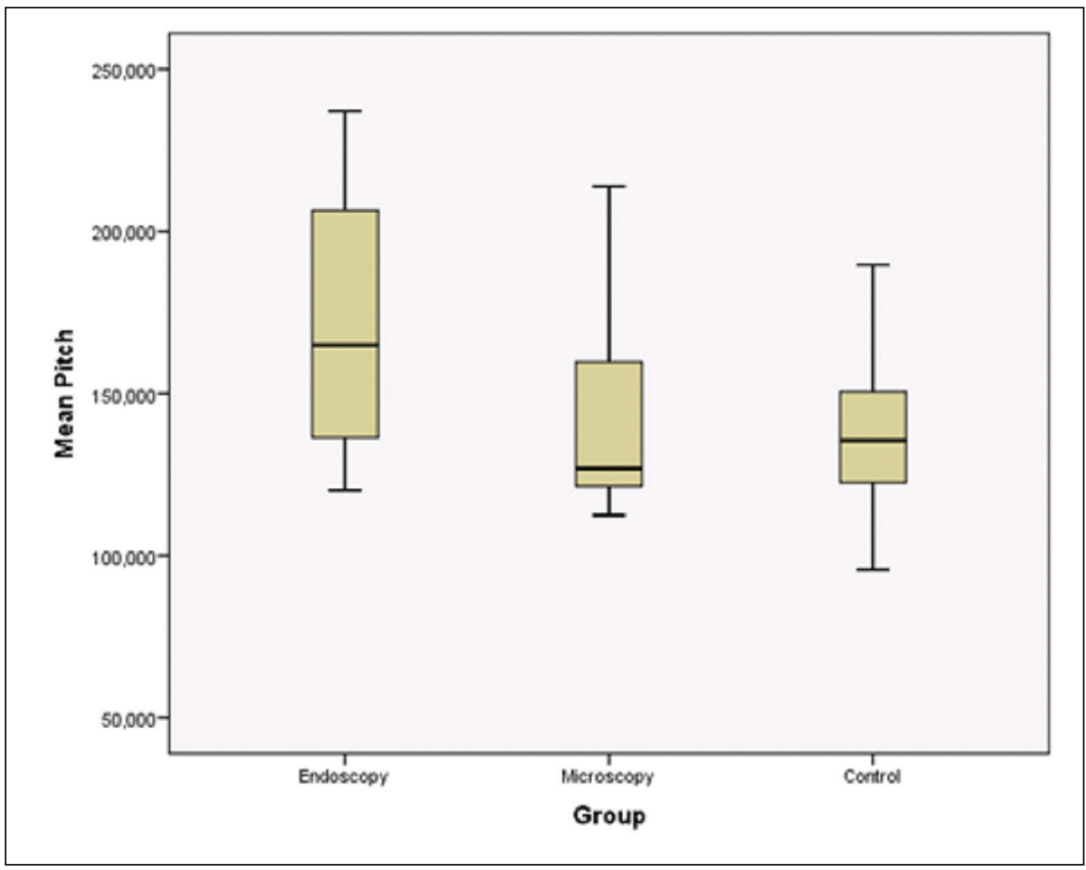

Figure 1: Comparison of F0 in three groups.

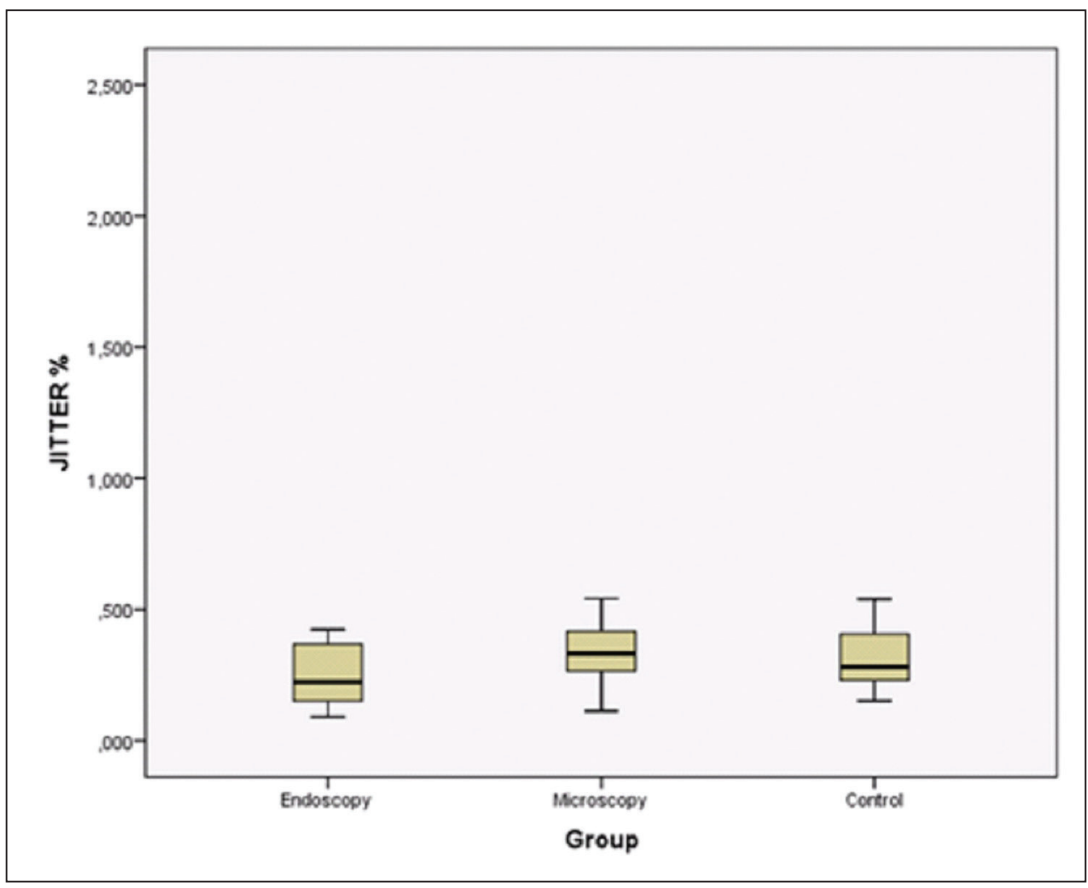

Figure 2: Comparison of jitter \% in three groups. 
Table II: Comparison of Objective Acoustic Parameters

\begin{tabular}{lccccccc}
\hline & \multicolumn{2}{c}{ Endoscopic Approach } & \multicolumn{2}{c}{ Microscopic Approach } & \multicolumn{3}{c}{ Control } \\
& Mean \pm SD & Median [Q1-Q3] & Mean \pm SD & Median [Q1-Q3] & Mean \pm SD & Median [Q1-Q3] & $\mathbf{p}$ \\
\hline Mean pitch & $170.66 \pm 37.88$ & $\begin{array}{c}164.96 \\
{[134.93-206.43]}\end{array}$ & $141.09 \pm 28.89$ & $\begin{array}{c}126.79 \\
{[121.31-159.86]}\end{array}$ & $137.03 \pm 20.94$ & {$[121.91-151.99]$} & $\mathbf{0 . 0 0 1}$ \\
\hline JITTER \% & $0.35 \pm 0.45$ & $0.22[0.15-0.37]$ & $0.40 \pm 0.24$ & $0.33[0.25-0.44]$ & $0.32 \pm 0.14$ & $0,28[0.23-0.41]$ & $\mathbf{0 . 0 3 6}$ \\
\hline JITTER PQQ5 & $0.19 \pm 0.24$ & $0.10[0.09-0.23]$ & $0.22 \pm 0.13$ & $0.18[0.13-0.27]$ & $0.19 \pm 0.08$ & $0.17[0.14-0.23]$ & $\mathbf{0 . 0 4 2}$ \\
\hline SCHIMMER \% & $1.99 \pm 1.67$ & $1.43[0.97-2.19]$ & $2.47 \pm 1.78$ & $1.95[1.23-3.09]$ & $1.81 \pm 1.00$ & $1.50[1.15-2.33]$ & 0.450 \\
\hline HNR (dB) & $20.93 \pm 6.19$ & $22.98[16.69-25.44]$ & $19.24 \pm 4.54$ & $20.32[14.78-22.85]$ & $20.90 \pm 3.76$ & $21.06[18.85-23.71]$ & 0.134 \\
\hline
\end{tabular}

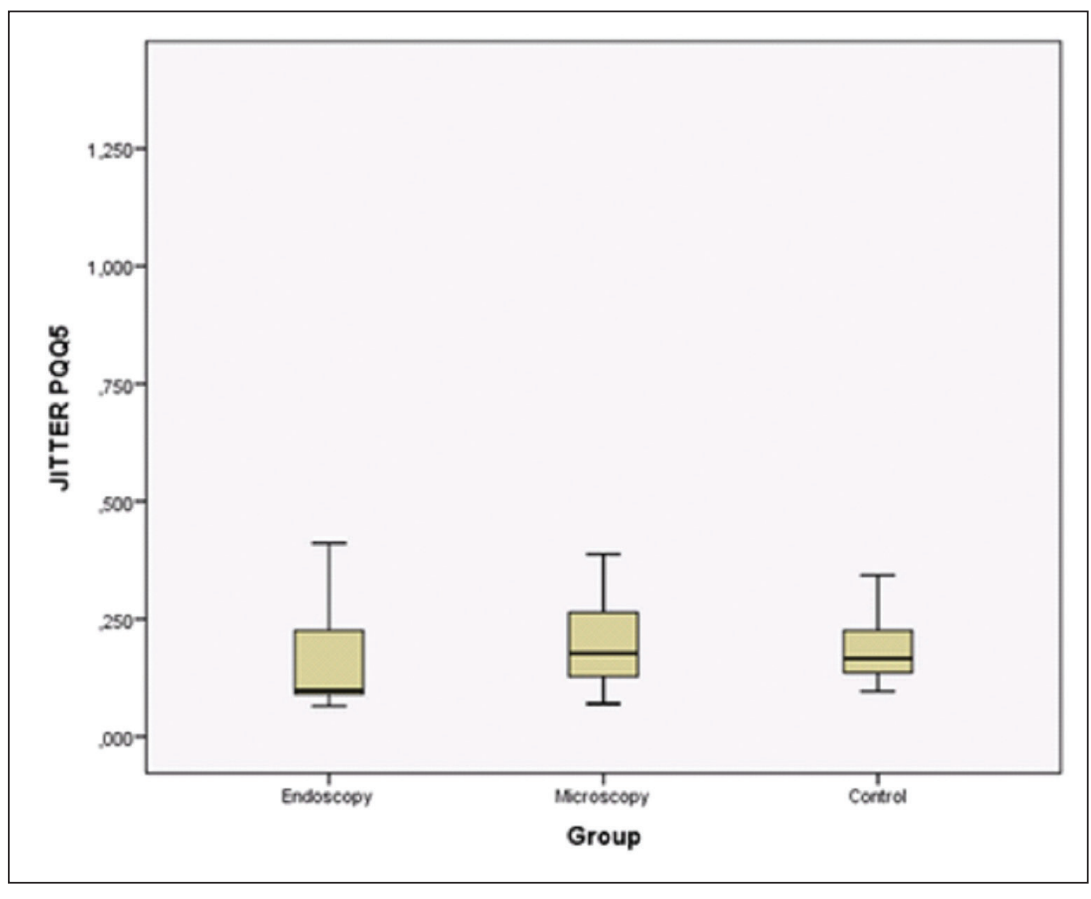

Figure 3: Comparison of jitter PQQ5 in three groups. complications, visualization of tumor and results of CSF rhinorrhea. We reckon our study of voice analysis is unique as we have not found any similar researches in the literature written in English language search that we conducted.

This study evaluates preoperative and postoperative voice analysis results and subjective voice parameters of 3 different groups of subjects. Due to the fact that VHI-30 is a long and a time-consuming questionnaire, a shorter Turkish version, named as VHI-10, was preferred. There was no statistically significant relationship in VHI-10 assessment scores.

Our objective voice analysis is an easily applicable, noninvasive method. Although there were many parameters, in assessment of voice analysis we used the following parameters; FO, which is the cycle vocal cords produce in a second; jitter, which shows the variability of the pitch voice; shimmer, which shows the variability from peak to peak; HNR is a measure that quantifies the amount of additive noise in the voice signal and also called Harmonics-to-Noise Ratio which is expressed in decibel. (If $99 \%$ of the energy of the signal is in the periodic part, and $1 \%$ is noise, the HNR is $10^{*} \log 10(99 / 1)$ $=20 \mathrm{~dB}$ ).

Jitter and shimmer are used to verify the level of instability (perturbation) in the voice quality and they are modestly correlated with voice quality characteristics such as hoarseness or roughness (7). Although we have not found any similar researches, there are many other studies over smokers. These studies present different results in both cigarette and e-cigarette smokers. In the study of Gonzales and Carpi, jitter and smoothed pitch perturbation quotient (SPPQ) were found significantly higher in smoking group (4). In the study of Tuhanioglu et al. jitter, shimmer and shimmer $\mathrm{dB}$ were reported high in smoking group (9).

In our study, jitter, jitter PQQ5 and shimmer \% was higher in $\mathrm{MO}$ group. This increase was statistically significant in jitter and jitter PQQ5 $(p=0.036)$. The increase was not statistically significant in shimmer \% $(p>0.05)$. 
F0 is the measurement of a person's voice frequency determining how high or low the person's voice is. It is the frequency of vocal fold vibration and it corelates with the vocal fold tension and changes in the rate of subglottic air pressure. Acar et al. reported that an increase was observed in F0 value in the postoperative period in patients with nasal polyposis (1). Atan et al. also reported that there was a significant increase in FO value in the postoperative period in patients with total septal deviation (2). F0 value increases in the recovery period of nasal cavity or when an operation is performed to restore anatomical structures. There was also a statistically significant difference in EO patients with respect to FO values in the present study.

HNR is the proportion of harmonic sound to noise in the voice measured in decibels. The lower the HNR, the more noise in the voice. Tafiadis et al. did not report a statistical difference between HNR values of smoker and non-smoker groups in their study (8). There was also no statistical difference within HNR values of groups in our study.

All of these researches seem to put endoscopic approach one step forward however our research was restricted by the width differences of ethmoidal and sphenoid sinuses that were opened during the interventions. Standardization was not possible with respect to their sizes.

\section{CONCLUSION}

Endoscopic surgical intervention is preferable among microscopic technique due to some evident advantages. We assessed the effects of endoscopic and microscopic techniques on individuals' voice quality. As a result; the difference between F0, jitter, jitter PQQ5 values reached statistical significance in patients who underwent endoscopic approach when compared to the values of microscopic intervention. The results of this study suggest that endoscopic method is better by means of vocal quality because it keeps the anatomically realistic model of sinuses and nasal cavity which provides resonance for the voice.

\section{REFERENCES}

1. Acar A, Cayonu M, Ozman M, Eryilmaz A: Changes in acoustic parameters of voice after endoscopic sinus surgery in patients with nasal polyposis. Indian J Otolaryngol Head Neck Surg 66(4):381-385, 2014

2. Atan D, Ozcan KM, Gurbuz AB, Dere H: The Effect of septoplasty on voice performance in patients with severe and mild nasal septal deviation. J Craniofac Surg 27(5):1162-1164, 2016

3. Gao Y, Zheng H, Xu S, Zheng Y, Wang Y, Jiang J, Zhong C: Endoscopic versus microscopic approach in pituitary surgery. J Craniofac Surg 27(2):157-159, 2016

4. Gonzalez J, Carpi A: Early effects of smoking on the voice: A multidimensional study. Med Sci Monit 10(12):649-656, 2004

5. Jankowski R, Auque J, Simon C, Marchal JC, Hepner H, Wayoff M: Endoscopic pituitary tumor surgery. Laryngoscope 102(2):198-202, 1992

6. Liu JK, Das K, Weiss MH, Laws ER Jr, Couldwell WT: The history and evolution of transsphenoidal surgery. J Neurosurg 95(6):1083-1096, 2001

7. Natour YS, Darawsheh WB, Bashiti S, Wari M, Taha J, Odeh T: A study of VHI scores and acoustic features in street vendors as occupational voice users. J Commun Disord 71:11-21, 2018

8. Tafiadis D, Toki El, Miller KJ, Ziavra N: Effects of early smoking habits on young adult female voices in Greece. J Voice 31(6):728-732, 2017

9. Tuhanioglu B, Erkan SO, Ozdas T, Derici C, Tuzun K, Senkal $\mathrm{OA}$ : The effect of electronic cigarettes on voice quality. J Voice S0892-1997(17):30623-30629, 2018 